\title{
Towards Socially Assistive Robotics
}

Adriana Tapus* and Maja J Matarić* *Interaction Lab., University of Southern California

\section{Introduction}

The recent trend toward developing a new generation of robots that are capable of moving and acting in human-centered environments, interacting with people, and participating in our daily lives has introduced the need for building robotic systems able to learn how to use their bodies to communicate and to react to their users in a social and engaging way. Social robots that interact with humans have thus become an important focus of robotics research.

The Human-Robot Interaction (HRI) for socially assistive robotics applications is a new, growing, and increasingly popular research area at the intersection of a number of fields, including robotics, medicine, psychology, ethology, neuroscience, and cognitive sciences. New applications for robots in health and education are being developed for broad populations of users. The world's population is growing older, thereby introducing a wide array of challenges. It is estimated that in 2040 there will be three times more people over age 85 than they are today. Many are expected to need physical and cognitive assistance. Even if nursing homes and other care facilities can provide assistance, space and staff shortages are already becoming an issue. As the elder population continues to grow, a great deal of attention and research will be dedicated to assistive systems that allow the elderly to live independently in their own homes. The first efforts towards having socially assistive robotic systems for the elderly have been focused towards constructing robot-pet companions aimed at reducing stress and depression [5] [11].

原稿受付 2006 年 4 月 21 日

キーワード: Assistive Robotics, Social Robotics, HumanRobot Interaction, Autonomous Robotics, Rehabilitation Robotics

*941 West 37th Place, RTH 407, Mailcode 0781, Los Angeles, CA 90089-0781, USA
In addition to the growing elderly population, other large user populations represent ideal beneficiaries of socially assistive robotics. Those include individuals with physical impairments and those in rehabilitation therapy, where socially assistive technology can serve to improve not only mobility [12] but also for outcomes in recovery. Finally, individuals with cognitive disabilities and developmental and social disorders (e.g., autism [2]) constitute another growing population that could benefit from assistive robotics in the contuxt of special education, therapy, and training.

In order to be able to aid the target user populations, an effective socially assistive robot must understand and interact with its environment, exhibit social behavior, and focus its attention and communication on the user in order to help the user achieve specific goals. Social behavior plays an important role in the assistance of people with special needs. We believe that an adaptive, reliable and user-friendly hands-off therapist robot that can provide an engaging and motivating customized therapy protocol to participants in laboratory, clinic, and ultimately, home environments, can establish a very complex and complete human-robot relationship. Therefore, such robots must be endowed with humanoriented interaction skills and capabilities to learn from us or to teach us, as well as to communicate with us and understand us.

Given these goals. socially assistive robotics poses multi-faceted challenges that require the consideration of the issues discussed in the next section.

\section{Challenges of Socially Assistive Robotics}

Socially assistive robotics is a very challenging domain in part because it involves interactions with vulnerable users, which involves a variety of sensitive ethical issues. To address the safety component of that spectrum, our work addresses a new niche: contact-free 
social robotic assistance. The physical embodiment of the robot plays a key role in its assistive effectiveness. Embodiment denotes not only physical reality but also the participative status. We focus on embodied interaction, because the physical and social world yields form, substance, and meaning from such interaction. The notion of embodiment represents a strong concept in the phenomenology of perception developed by Maurice Merleau-Ponty [8]. Three different meanings of embodiment have been identified in Merleau-Ponty's work:

- The physical embodiment of a human subject, with legs and arms, and of a certain size and shape;

- The set of bodily skills and situational responses that humans have developed;

- The cultural skills abilities and understandings that humans responsively gain from the cultural world in which they are embedded.

All these aspects must be considered when creating an embodied robotic system. Nevertheless, it is well established that people attribute intentions, goals, emotions, and personalities to even the simplest of machines with life-like movement or form [9]. Because of this combination of properties, embodiment constitutes a key means of establishing human-robot interaction, and specifically of getting the user to respond to the robot and become engaged in an interaction with it. Efforts in this research area have already been made (e.g., [1] [7]). However, social robotics has not yet tackled the complex challenges of assistive robotics where the overall goal is to achieve measurable progress toward the prescribed health, education, or training goals. Socially assistive robotics, our field of research focus, presents a new paradox: the goal of retaining user engagement can be in conflict with the health/training/education goals. For instance, it has been shown that human nurses that are most liked by patients are not the ones most effective in facilitating patient recovery. We believe that the robot's physical embodiment, its physical presence, and its shared context with the user will play important roles in time-extended, sustained, goal-driven interactions in assistive domains.

Verbal and non-verbal communication provide social cues that make the robots appear more "intuitive and natural", and increase the probability of humans to create strong bonds with the robots such as those formed with other humans. We posit that understanding human affect and reacting to behave more suitably to dif- ferent social situations (e.g., so as to avoid misunderstandings and to permit for more natural interactions) and to to it appropriately, can help the robots improve the task performance.

Humans differentiate readily among social stimuli such as faces, voices, and gestures. Consequently, physical appearance and verbal and non-verbal communication are important components of human-robot interaction. It has already been shown that physical appearance must be appropriately matched to the robot's cognitive and interactive capabilities in order to avoid unmet user expectations. It is still a great challenge to create robots that can behave in a fashion that is socially similar to people. Therefore, the more humanlike the robot appears, the higher the expectations of people interacting with it are. Since our research area focuses on assistive applications, we are more interested in the believability of our systems rather than in their realism. This philosophy is based on research and observation of human interaction. Youthful appearance and other anthropomorphic but not highly realistic appearance are likely particularly suitable for assistive domains. Toward that end, our therapist robot, shown in Fig. 1, resembles a youthful humanoid. We have successfully used this and other, simpler mobile robots, to validate prototype therapist systems that assist, encourage and socially interact with users in the process of cardiac convalescence, stroke rehabilitation, and education [3] [4] [6] [10].

Since this work is directed toward assistive applications, emotional and appraisal support involving empathy also plays a crucial role in our research. The role of user personality and empaty have not yet been studied in the context of socially assistive robotics, and are one

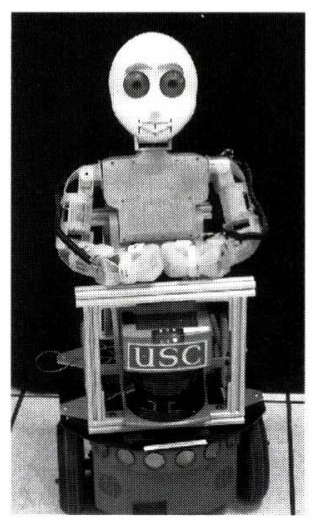

Fig. 1 Bandit: our humanoid therapist robot 
of our areas of research focus.

\section{Conclusions}

Our research and results to date into socially assistive robotics show the promises of this new research area and extend the horizons of the field of robotics. Our ongoing research is aimed at addressing and solving the above mentioned issues related to embodiment, social behaviors, and empathy, at developing effective embodied assistive systems, and at extending our understanding of human social behavior towards assistive applications.

\section{References}

[1] C. Breazeal: Designing Sociable Robots. MIT Press, 2002.

[ 2 ] K. Dautenhahn, I. Werry, J. Rae, P. Dickerson, P. Stribling and B. Ogden, Robotic Playmates: 'Analysing Interactive Competencies of Children with Autism Playing with a Mobile Robot,' Socially Intelligent Agents: Creating Relationships with Computers and Robots. pp.117-124, Kluwer Academic Publishers, 2002.

[3] J. Eriksson and M.J. Matarić: "Hands-Off Assistive Robotics for Post-Stroke Arm Rehabilitation," Proceedings IEEE International Conference on Rehabilitation Robotics (ICORR-05),
2005

[4] D. Feil-Seifer and M.J. Matarić: "Socially Assistive Robotics," Proceedings of IEEE International Conference on Rehabilitation Robotics (ICORR-05), 2005.

[5] T. Fong, I. Nourbakhsh and K. Dautenhahn: "A survey of socially interactive robots," Robotics and Autonomous Systems, vol.42, no.3-4, pp.143-166, 2003.

[6] R. Gockley and M.J. Matarić: "Encouraging Physical Therapy Compliance with a Hands-Off Mobile Robot," Proceedings of the First International Conference on Human Robot Interaction (HRI-06), pp.150-155, 2006.

[ 7 ] S. Kiesler and J. Goetz: "Mental Models and Cooperation with Robotic Assistants," Proceedings of Conference on Human Factors in Computing Systems (CHI), pp.576-577, 2002.

[8] M. Merleau-Ponty: Phenomenology of Perception. Tr. G. Smith. 1962.

[ 9 ] B. Reeves and C. Nass: The Media Equation: How People Treat Computers, Television, and New Media Like Real People and Places. Cambridge University Press, 1998.

[10] A. Tapus and M.J. Matarić: "Introvert vs. Extrovert HandsOff Robot for Post-Stroke Rehabilitation Therapy," submitted to The 10th International Symposium of Experimental Robotics (ISER-06), 2006.

[11] M. Walton: Meet PARO, The Therapeutic Robot Seal. CNN, 2003.

[12] H.A. Yanco: 'Wheelesley, A Robotic Wheelchair System: Indoor Navigation and User Interface,' Lecture Notes in Artificial Intelligence: Assistive Technology and Artificial Intelligence. pp.256-268, Springer-Verlag, 1998.

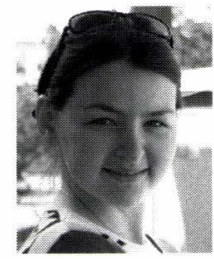

\section{Adriana Tapus}

Adriana Tapus is a postdoctoral fellow at Interaction Lab, Computer Science Department, University of Southern California, USA. She received her M.S. in Computer Science at University Joseph Fourier, Grenoble, France in 2002 and her degree of Engineer in Computer Science and Engineering at Politechnica University of Bucharest, Romania in 2001. She received her Ph.D. degree from the Swiss Federal Institute of Technology, Lausanne (EPFL) in 2005. Her main supervisor was Prof. Roland Siegwart. Her dissertation pertained to finding a natural solution to do human-like navigation using fingerprints of places. Her current research interests include socially assistive robotics, human-robot interaction, humanoid robotics, machine learning, and computer vision.

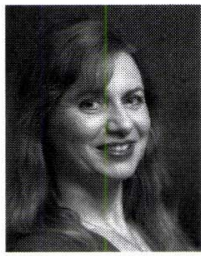

\section{Maja J Matarić}

Maja J Matarić is an associate professor in the Computer Science Department and the Neuroscience Program at the University of Southern California, Founding Director of the USC Center for Robotics and Embedded Systems, and Co-Director of the USC Robotics Research Lab. She received her Ph.D. in Computer Science and Artificial Intelligence from MIT in 1994, her M.S. in Computer Science from MIT in 1990, and her B.S. in Computer Science from the University of Kansas in 1987. She is a recipient of the NSF Career Award, the IEEE Robotics and Automation Society Early Career Award, the MIT TR100 Innovation Award, and the USC School of Engineering Junior Research Award. Her research is aimed at endowing robots with the ability to help people and involves systems ranging from individual assistants (for convalescence, rehabilitation, training, education) to cooperative robot teams and problems of intelligent control and learning in complex, high dimensional/high degree of freedom systems that integrate perception, representation, and interaction with people. Research details are found at http://robotics.usc.edu/interaction/ 